\title{
Continuously Degrading Resource and Interval Dependent Activity Durations in Nuclear Medicine Patient Scheduling
}

\author{
Cyrille Dejemeppe and Yves Deville \\ ICTEAM, Université Catholique de Louvain (UCLouvain), Belgium, \\ $\{$ cyrille.dejemeppe, yves.deville\}@uclouvain.be
}

\begin{abstract}
Nuclear Medicine (NM) is a medical imaging technique in which patients are administered radioactive tracers. As the tracers decay in the human body, they emit photons, which are then captured to generate an image used for diagnostic purposes. The schedule of daily patients in an NM center is a hard and interesting problem due to the continuous decay of these radioactive tracers. This scheduling problem allows us to define two new scheduling concepts: continuously degrading resources and interval dependent activity durations. In this paper, we model the NM scheduling problem as a COP; we propose a resolution strategy using Constraint Programming with LNS, and we introduce a new propagator to deal with continuously degrading resources and interval dependent activity durations.
\end{abstract}

\section{Introduction}

Nuclear Medicine is a clinical practice in which patients are administered nuclear tracers in order to provide diagnostic information for a wide range of diseases. A nuclear tracer is a set of radioactive compounds. As defined in [3], the activity of a radioactive tracer decreases with time according to the following law of decay:

$$
\operatorname{Rad}(t)=\operatorname{Rad}_{0} \times \mathrm{e}^{\frac{-t \ln (2)}{t_{0.5}}}
$$

where $\operatorname{Rad}_{0}$ is the initial activity of the decaying substance and $t_{0.5}$ is its half-life. As it decays, the radioactive component in the tracer emits gamma rays or high-energy photons. The energy levels of these emissions are such that a significant amount of energy can exit the body without being scattered or attenuated. External gamma-ray sensors allow capturing these emissions, and computers are then able to recreate an image from them. As stated in [2], NM has several advantages over other medical imaging techniques. The precision and the quality of the images obtained with NM makes it a technique widely used for medical imaging.

Nuclear Medicine Problem (NMP) consists in the optimization of the schedule of patient workflows inside an NM center. Patients coming to an NM center are first administered a nuclear tracer. Then they have to wait in a waiting room in order to allow their body to incorporate the tracer. The duration of this waiting time has to last a minimum amount of time, otherwise the human body will not have fully incorporated the nuclear tracer. On the other hand, the waiting time cannot be too long, since then the radioactive tracer would have decayed for too long, leading to its radioactive activity's being too low to provide satisfactory images. Once a patient's body has fully incorporated the tracer, the patient goes into a scanning room in which the image is captured. 
There can be several different scanning rooms which differ in their scanner equipment. The amount of time needed by the scanner equipment to capture the image directly depends on the amount of time the patient has been waiting after having been administered the radioactive tracer.

Even if the workflows of the patients are similar, they can differ by the duration of each step inside the workflow, by the type and quantity of tracer administered, by the minimal and maximal durations of their waiting times, and by the scanning room and equipment needed to capture the image. As stated in [7], the acquisition time can be expressed as a linear function of the waiting time as follows:

$$
\text { acquisition time }=\alpha+\delta \times \text { waiting time }
$$

where $\alpha$ and $\delta$ are positive constants depending on the quantity and type of tracer administered to the patient. Figure $1 \mathrm{~b}$ shows an example of how the acquisition time depends on the waiting time.

Patients are administered their tracer dose with a special injector and most NM centers only have a few of these. The scanning rooms exist in different instances and sometimes they have different kinds of equipment. There are not very many of these scanning rooms, and some NM centers even have only one scanning room. The day's radioactive tracers are delivered to the NM center every morning. These tracers decay with time, i.e. their radioactive activity decreases. As the patients need to be injected with a certain amount of radioactivity, the quantity of tracer they are injected with increases with time to compensate for the decrease in the radioactivity of the tracer that has occurred since the beginning of the day. An example of the radioactive activity of the widely used Fludeoxyglucose (FDG) tracer over time can be seen in Figure 1a.

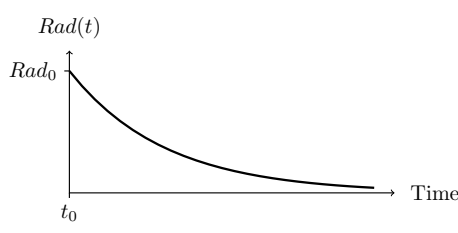

(a) Activity of the FDG tracer over time

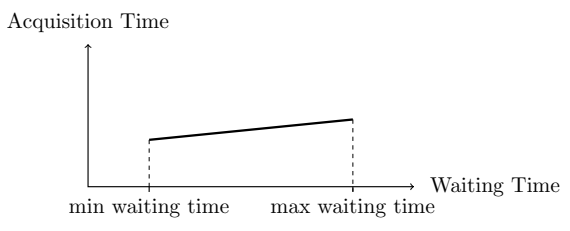

(b) Time required for image acquisition in function of the length of the waiting time after injection

Fig. 1: Example of radioactivity decrease and its effects on acquisition time

NMP is an optimization problem with two distinct objectives. First, the purpose is to be able to treat patients in the shortest amount of time possible. This would possibly allow treating more patients per day. The second objective is to minimize the total consumption of radioactive tracer. Indeed, these substances are very expensive and being able to start the day with a smaller quantity would save money. These objectives are not aggregated, and NMP is thus a bi-objective optimization problem.

We model NMP as a scheduling problem. To model the decay of nuclear tracers, we have to introduce two new scheduling concepts: continuously degrading resources and interval dependent activity durations. The abstraction of a continuously degrading resource allows us to model a resource whose capacity decreases continuously over time. 
This allows us to represent the decay of the radioactive tracers, said capacity representing the decreasing radioactivity of the tracer. The abstraction of an interval dependent activity is used to model an activity whose duration depends on the interval between the activity itself and the activity preceding it. As we introduce these two scheduling abstractions, we define new constraints and their associated new propagators.

The two new abstractions we introduce could be used in several other scheduling applications. The continuously degrading resource abstraction could be used to model chemical compounds which react with each other and whose quantities thus continuously decrease over time. The interval dependent activity duration abstraction could be used to model other medical processes such as in the cure of an uncoupled shoulder: if the time between the radiography and the replacement of the shoulder is longer, the muscles will tighten more, so that the operation to replace the shoulder will take more time. This article also intends to illustrate how to solve cumulative scheduling problems with business constraints. As such, the modelling and search techniques described for NMP are as generic as possible.

\section{The Model}

In this section, we define a model for NMP defined in Section 1. NMP is a scheduling problem and can thus be defined by the four main components of a scheduling problem: activities, resources, constraints and objectives.

The activities of our model are the steps of the patient workflows. The workflow for each patient consists of a job $j$ containing two activities: injection and image acquisition. The resources of NMP are the injectors and the scanning rooms, which are modelled by the cumulative resources, and the radioactive tracers, which are modelled by a new abstraction: a continuously degrading resource. There are precedence constraints imposing an order between the activities of the same job. There are also resource constraints to ensure that the resource capacities are not exceeded. Some constraints must also be imposed to ensure that the waiting time of a patient between the injection and the image acquisition remains bounded. Finally, some constraints are needed to model the durations of the activities: a fixed constraint for the injection, but one that depends on the length of the waiting time for the image acquisition. The objective of treating a patient in as little time as needed is modelled by a makespan minimization. On the other hand, the minimization of the total consumption of radioactive tracers is modelled by the sum of the consumptions of the tracer by the patients. We express our scheduling model more formally as a Constraint Optimization Problem (COP) $\langle X, D, C, O\rangle$ as follows.

$X$ : The Set of Variables The variables of our problem are the starting and ending times of each activity. We consider instances containing $k$ jobs and inside each job there are $n$ activities. So for each activity $A_{i, j}$, the $j^{\text {th }}$ activity of the $i^{t h}$ job, we define two decision variables: $\operatorname{start}\left(A_{i, j}\right)$ and $\operatorname{end}\left(A_{i, j}\right)$ with $1 \leq i \leq k$ and $1 \leq j \leq n$.

$D$ : The Domains of the Variables The start variables $\operatorname{start}\left(A_{i, j}\right)$ of the activities have the following domains:

$$
D\left(\operatorname{start}\left(A_{i, j}\right)\right)=\left\{\begin{array}{l}
{\left[0 ; \text { horizon }- \text { duration }\left(A_{i, j}\right)\right] \text { if the duration is fixed }} \\
{[0 ; \text { horizon }] \text { otherwise }}
\end{array}\right.
$$


with $1 \leq i \leq k, 1 \leq j \leq n$, and horizon being the horizon of the problem.

Similarly, the end variables end $\left(A_{i, j}\right)$ of the activities have the following domains:

$$
D\left(\operatorname{end}\left(A_{i, j}\right)\right)=\left\{\begin{array}{l}
{\left[\text { duration }\left(A_{i, j}\right) ; \text { horizon }\right] \text { if the duration is fixed }} \\
{[0 ; \text { horizon }] \text { otherwise }}
\end{array}\right.
$$

with $1 \leq i \leq k$ and $1 \leq j \leq n$.

$C$ : The Constraints The first constraints described here define an order between the activities belonging to the same job and impose a setup time between two successive activities when needed:

$$
\operatorname{end}\left(A_{i, j}\right)+\operatorname{setup}\left(A_{i, j}, A_{i, j+1}\right) \leq \operatorname{start}\left(A_{i, j+1}\right)
$$

with $1 \leq i \leq k, 1 \leq j \leq n-1$ and $\operatorname{setup}\left(A_{i, j}, A_{i, j+1}\right)$ being the amount of time needed between the two activities. If the two successive activities are not subject to a setup time, then we have $\operatorname{setup}\left(A_{i, j}, A_{i, j+1}\right)=0$.

Another constraint must impose a maximal delay between those pairs of activities which need it.

$$
\operatorname{start}\left(A_{i, j+1}\right)-\operatorname{end}\left(A_{i, j}\right) \leq \text { delay }_{\max }\left(A_{i, j}, A_{i, j+1}\right)
$$

with $1 \leq i \leq k, 1 \leq j \leq n-1$, and delaymax $\left(A_{i, j}, A_{i, j+1}\right)$ being the maximal amount of time allowed between the two activities. If the two successive activities are not subject to a maximum delay, then we have delay $_{\max }\left(A_{i, j}, A_{i, j+1}\right)=$ horizon.

As explained above, some activities have fixed durations while others have interval dependent durations. In our model, some durations depend linearly on the length of the interval according to a monotonic increasing function $f($ interval $)=\alpha+\delta \times$ interval where $\alpha$ and $\beta$ are positive constants. The following constraints explain these properties:

$$
\operatorname{end}\left(A_{i, j}\right)-\operatorname{start}\left(A_{i, j}\right)= \begin{cases}\operatorname{duration}\left(A_{i, j}\right) & \text { if fixed duration } \\ f\left(\operatorname{start}\left(A_{i, j}\right)-\operatorname{end}\left(A_{i, j-1}\right)\right) & \text { otherwise }\end{cases}
$$

with $1 \leq i \leq k, 1 \leq j \leq n-1$, and $A_{i, j}$ being an activity with interval dependent duration.

The cumulative resource constraints can be expressed as follows:

$$
\sum_{A} \operatorname{usage}\left(A, R_{\text {cum }}, t\right) \leq \operatorname{capacity}\left(R_{\text {cum }}\right)
$$

where $0 \leq t \leq$ horizon, $R_{\text {cum }}$ is a cumulative resource, usage $\left(A, R_{\text {cum }}, t\right)$ is the usage of $R_{\text {cum }}$ by the activity $A$ at time $t$, and capacity $\left(R_{\text {cum }}\right)$ is the capacity of $R_{\text {cum }}$.

Finally, we have to express the constraint on the continuously degrading resources. We model these constraints not by diminishing the resource capacity over time, but by increasing the quantity required by the activities over time. We assume that the needed amount of a resource for an activity is consumed at its starting time in an atomic way. The quantity needed by an activity $A_{i, j}$ of the continuously degrading resource $R_{d e c}$ if starting at a time $t$ is

$$
q\left(A_{i, j}, R_{d e c}, t\right)=\frac{C_{i}}{R a d(t)}=\frac{C_{i}}{R a d_{0}} \times \mathrm{e}^{\frac{t \ln (2)}{t_{0.5}}}
$$


where $\operatorname{Rad}(t)$ is defined in Equation $1, \operatorname{Rad}_{0}$ and $t_{0.5}$ depend on $R_{d e c}$, and $C_{i}$ is a positive constant allowing to determine the amount of radioactivity needed by patient corresponding to job $i$. This formula expresses that the quantity of tracer a patient is injected is inversely proportional to the radioactivity of the tracer. We must ensure that the total consumption of each resource is less than or equal to the initial capacity of that resource. This constraint is expressed by

$$
\sum_{A} q\left(A, R_{\text {dec }}, \operatorname{start}(A)\right) \leq \text { initialCapacity }\left(R_{\text {dec }}\right)
$$

where initialCapacity $\left(R_{d e c}\right)$ is the initial capacity of the resource $R_{d e c}$.

$O$ : The Objectives The first objective is to minimize the makespan. This can be represented as follows:

$$
\text { minimize makespan }=\max _{A}(\text { end }(A)) .
$$

The second objective is to minimize the total consumption of the continuously degrading resources. This can be expressed as follows:

$$
\operatorname{minimize} \sum_{R_{d e c}} \sum_{A} w_{d e c} \times q\left(A, R_{d e c}, \operatorname{start}(A)\right)
$$

where the $w_{\text {dec }}$ are positive weights.

\section{Propagation}

In this section, we describe how to perform propagation for the continuously degrading resource constraint and the interval dependent activity duration constraint.

\subsection{Continuously Degrading Resource}

The propagation procedure we describe achieves Bound Consistency (BC) for the constraint stated in Equation 10. For a given activity $A_{i, j}$ and a given continuously decreasing resource $R_{d e c}$, the quantity of resource required by the activity is a monotonic function increasing with time $t: q\left(A_{i, j}, R_{d e c}, t\right)$. This increasing quantity can be defined for NMP by Equation 1. The quantity of tracer a patient is injected is inversely proportional to the radioactivity of a tracer. We can thus express $q\left(A_{i, j}, R_{d e c}, t\right)$ as follows:

$$
q\left(A_{i, j}, R_{d e c}, t\right)=\beta_{i, j} \times \mathrm{e}^{\frac{t \ln (2)}{\gamma_{d e c}}}
$$

where $\beta_{i, j}$ is a constant depending on $A_{i, j}$ and $\gamma_{d e c}$ is a constant depending on $R_{d e c}$. We are thus able to rewrite the constraint stated in Equation 10 as follows:

$$
\sum \beta_{i, j} \times \mathrm{e}^{\frac{\operatorname{start}\left(A_{i, j}\right) \ln (2)}{\gamma_{\text {dec }}}} \leq \text { initialCapacity }\left(R_{\text {dec }}\right)
$$

To express this constraint as a linear sum constraint, we will use the view-based propagator derivation technique proposed in [6]. Given a propagator $p$, a view is represented by two functions $\phi$ and $\phi^{-1}$ that are composed with $p$ to obtain the desired propagator $\phi \circ p \circ \phi^{-1}$. The $\phi$ function transforms the input domain and $\phi^{-1}$ applies the inverse transformation to the propagator's output domain. To be able to use a linear bounded 
sum constraint propagator, we define a $\phi_{i, j}$ function for each variable $\operatorname{start}\left(A_{i, j}\right)$ involved in Equation 14 as follows:

$$
\phi_{i, j}(v)=\beta_{i, j} \times \mathrm{e}^{\frac{v \ln (2)}{\gamma_{d e c}}}
$$

The inverse functions $\phi_{i, j}^{-1}$ are defined as follows:

$$
\phi_{i, j}^{-1}(v)=\frac{\gamma_{d e c}}{\ln (2)} \times \ln \left(\frac{v}{\beta_{i, j}}\right)
$$

As values returned by the $\phi$ function are real values and our start variables only accept integer values, we consider the domain of $\operatorname{start}\left(A_{i, j}\right)$ mapped by $\phi$ is a discrete domain in which each value correspond to a single value in the domain of $\operatorname{start}\left(A_{i, j}\right)$. The definitions of $\phi$ and $\phi^{-1}$ allow us to use a classical linear sum constraint propagator as proposed in [1].

\subsection{Interval Dependent Activity Durations}

The propagation procedure we describe achieves $\mathrm{BC}$ for the constraint stated in Equation 7. As stated earlier, the $f$ function in Equation 7 is a linear monotonic increasing function. From this, we can rewrite the constraint depicted in Equation 7 as follows:

$$
\operatorname{end}\left(A_{i, j}\right)-\operatorname{start}\left(A_{i, j}\right)=\varepsilon_{i, j}+\delta_{i, j} \times\left(\operatorname{start}\left(A_{i, j}\right)-\operatorname{end}\left(A_{i, j-1}\right)\right)
$$

where $\delta_{i, j}$ and $\varepsilon_{i, j}$ are positive constants. Similarly to what was described in Section 3.1, by using trivial views for variables, we are able to obtain a classical linear sum equality constraint. The propagation of the interval dependent activity duration can be achieved by using the propagator of a linear sum equality constraint and views.

\section{Experimental Results}

To give an overview of the complex nature of the NM problem, we propose to solve four different versions of the problem. Each version adds a new source of complexity to the previous version. These problems are solved using a Constraint Programming $(\mathrm{CP})$ with Large Neighborhood Search (LNS) where the branching heuristic used is a binary first fail on the start variables. As defined in [8], a CP with LNS search favors the exploration of the search space at the loss of completeness. When a solution is found, several relaxations are applied to it and a new search begins from the partial solution obtained. For each version of our problem, a time limit of three minutes is imposed and the best values found for both objective functions (makespan and quantity of tracer consumed) are reported in Table 1. All experiments were conducted with the OscaR open-source solver [4]. The instances considered are lists from 10 to 50 patients obtained by a biased random generator we designed. The durations of patient activities and the resource capacities and decay parameters are generated using realistic values. However, typical NM centers with the considered configurations treat at most 25 patients per day and larger instances are considered to test the limits of the model.

The first problem, $V_{1}$, is a relaxation of the NM problem in which nor continuously decreasing resources nor the interval dependent activity durations (i.e. durations are fixed for all activities) are considered and the only objective is the minimization of the 
makespan. Hence, $V_{1}$ is a classical Cumulative Job-Shop Problem. In Table 1, we can observe that the quantity of tracer used (TQ) increases dramatically with the number of patients and the makespan (MS). This is due to the exponential nature of the quantity of tracer required with time by patients, as stated in Equation 9.

The second problem, $V_{2}$, adds the interval dependent activity duration constraint to the $V_{1}$ version of our problem. Hence, the durations of the acquisition activities of patients are not fixed any more and we add the constraint stated in Equation 7. Again, we only consider minimization of the makespan. When comparing the results from Table 1 for the problem $V_{1}$ with results for problem $V_{2}$, we can see the makespan and quantity of resource used are higher for $V_{2}$ than for $V_{1}$. This can be explained by two main reasons. First, the solutions for problem $V_{1}$ are not solutions for problem $V_{2}$. Indeed, $V_{2}$ adds a relation linking the waiting time of patients with the duration of imagery acquisition durations. This relation could not be respected in a solution for problem $V_{1}$. Second, as $V_{2}$ relaxes the duration of acquisition activities, the search space is larger for $V_{2}$ than for $V_{1}$. As both problems $V_{1}$ and $V_{2}$ have ran under the same conditions and with the same branching heuristics, it is normal that $V_{2}$ obtains solutions as good as those for $V_{1}$.

The third problem, $V_{3}$, adds the continuously decreasing resource constraint to the $V_{2}$ version of our problem. Furthermore, the search now focuses on minimizing the quantity of tracer used. As expected, when comparing results for problem versions $V_{2}$ and $V_{3}$ in Table 1, we observe that the quantity of tracer used is on average lower for $V_{3}$ than for $V_{2}$ as opposed to the makespan which is higher. To obtain solutions which are tradeoff between the two objective function, it is interesting to consider a bi-objective search version of our problem.

The fourth problem, $V_{4}$, considers the NM problem as a bi-objective problem minimizing the makespan and the quantity of tracer used. Hence, this version will find a set of non-dominated solutions instead of a single one. To solve this problem we use a variant of the constraint introduced in [5]. Results for the version $V_{4}$ of the problem are reported in Table 1. These results are the average best solutions obtained for both objectives. We can observe the reported average of the best solutions obtained are between the best and the worst values found for $V_{2}$ and $V_{3}$ for the makespan and the quantity of tracer used. In Figure 2, we report the Pareto front obtained by $V_{4}$ as well as the best solutions obtained by $V_{2}$ and $V 3$ on instances with 20 and 40 patients. We observe that some solutions obtained by $V_{4}$ are dominated by the best solutions obtained by $V_{2}$ and $V_{3}$. Nevertheless some other solutions are not dominated by best solution for $V_{2}$ nor for $V_{3}$. As such, the problem version $V_{4}$ is well suited to obtain a set of tradeoffs between the two objectives considered.

\section{Conclusion}

In this paper, we have described the NM problem and we modelled it as a scheduling problem. To deal with some characteristics of the problem, we introduced two new scheduling abstractions: continuously decreasing resources and interval dependent activity durations. These two scheduling abstractions were modelled with the help of views and sum constraints. Finally, we proposed an efficient method to solve the NM problem with CP and LNS. The resolution strategies and the problem tackled was declined in different versions. Each version allows to solve the problem according to the 


\begin{tabular}{|c|c|c|c|c|c|c|c|c|c|c|}
\hline \multirow[t]{2}{*}{ Problem Version } & \multicolumn{2}{|c|}{10 Patients } & \multicolumn{2}{|c|}{20 Patients } & \multicolumn{2}{|c|}{30 Patients } & \multicolumn{2}{|c|}{40 Patients } & \multicolumn{2}{|c|}{50 Patients } \\
\hline & MS & TQ & MS & TQ & MS & TQ & MS & TQ & MS & TQ \\
\hline Problem $V_{1}$ & 251 & 9.97 & 446 & 40.44 & 650 & 129.9 & 867 & 516 & 1,048 & 1,268 \\
\hline Problem $V_{2}$ & 253 & 11.49 & 486 & 51.16 & 737 & 242.6 & 994 & 1,065 & 1,211 & 2,770 \\
\hline Problem $V_{3}$ & 291 & 9.17 & 530 & 39.04 & 779 & 164.7 & 1,029 & 671 & 1,245 & 1,862 \\
\hline Problem $V_{4}$ & 266 & 9.42 & 495 & 38.32 & 751 & 182.2 & 1,011 & 757 & 1,234 & 1,885 \\
\hline
\end{tabular}

Table 1: Average objective values for different versions of problem on different size of instances. MS is the makespan and TQ is the quantity of tracer used. For problems $V_{1}, V_{2}$ and $V 3$, the values reported are the average values for the instance size considered. For problem $V_{4}$, values reported are the averages of the best values found for each objective for the instance size considered.

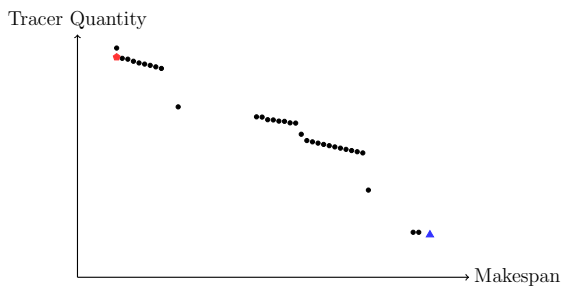

(a) Pareto front of an instance with 20 patients

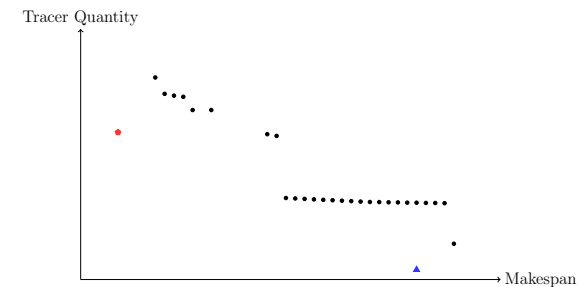

(b) Pareto front of an instance with 40 patients

Fig. 2: Comparison of Pareto front solutions obtained by the problem version $V_{4}$ and the best solutions obtained by versions $V_{2}$ and $V_{3}$. The red squares are the best solutions obtained by version $V_{2}$, the blue triangles are the best solutions obtained by $V_{3}$ and the black circles are the points of the Pareto front obtained by the version $V_{4}$.

desired objective function or to perform bi-objective optimization to obtain a set of solutions which are tradeoffs between these objectives.

The proposed modelling and search techniques are generic and could be used for other cumulative scheduling problems with specific constraints. The only requirement is that these specific constraints combine existing constraints (i.e. with an existing propagator) on new variables which are defined as functions of variables of the initial problem (e.g. start and end activity variables). Thanks to the use of views, propagators of these constraints can be applied. Our approach allows a large range of cumulative scheduling problems with specific additional constraints.

Acknowledgments The authors want to thank the anonymous reviewers for their helpful comments. This research is supported by the Mirror Project, the FRFC project 2.4504.10 of the Belgian FNRS, and the UCLouvain Action de Recherche Concerte ICTM22C1. 


\section{References}

1. K. R. Apt. Principles of Constraint Programming. Cambridge University Press, UK, 2003.

2. S. Cherry, J. Sorenson, and M. Phelps. Physics in Nuclear Medicine. Elsevier Health Sciences, 2012.

3. J. S. Fowler and T. Ido. Initial and subsequent approach for the synthesis of $18 \mathrm{fdg}$. Seminars in Nuclear Medicine, 32(1):6 - 12, 2002. Impact of FDG-PET Imaging on the Practice of Medicine.

4. OscaR Team. OscaR: Scala in OR, $2012 . \quad$ Available from https://bitbucket.org/oscarlib/oscar.

5. P. Schaus and R. Hartert. Multi-objective large neighborhood search. In Principles and Practice of Constraint Programming, pages 611-627. Springer Berlin Heidelberg, 2013.

6. C. Schulte and P. J. Stuckey. Efficient constraint propagation engines. Transactions on Programming Languages and Systems, 31(1):2:1-2:43, Dec. 2008.

7. H. Schder, Y. Erdi, S. Larson, and H. Yeung. Pet/ct: a new imaging technology in nuclear medicine. European Journal of Nuclear Medicine and Molecular Imaging, 30(10):14191437, 2003.

8. P. Shaw. Using constraint programming and local search methods to solve vehicle routing problems. In Principles and Practice of Constraint ProgrammingCP98, pages 417-431. Springer, 1998. 